\title{
New treatments in membranous glomerulopathy - from the pitfalls of rituximab to a new era of biological treatments
}

Filipa Ferreira, Ana Teresa Nunes

Nephrology department, Centro Hospitalar de São João - Porto, Portugal

\section{ABSTRACT}

Primary membranous nephropathy (PMN) is the main cause of nephrotic syndrome in adults. The recognition that this kidney-specific disease is the result of an autoimmune process has changed diagnostic and therapeutic approaches. The determination of anti-phospholipase A2 receptor and thrombospondin type- 1 domain containing $7 \mathrm{~A}$, when available, are part of the diagnostic and therapeutic monitoring workup. More recently, more putative antigens have been discovered.

Treatment of PMN relies on optimal supportive care but immunosuppression is indicated in patients at risk of progressive kidney injury. Immunosuppression schemes commonly used are cyclophosphamide/steroids (modified Ponticelli), calcineurin inhibitors/steroids and, after the MENTOR trial, rituximab has also been considered a first-line agent in non-severe cases. However, even in the MENTOR trial, $40 \%$ of patients did not achieve remission.

Rituximab-resistant PMN cases have been published. Many mechanisms have been implicated in rituximab resistance, such as the development of anti-drug antibodies, interindividual variability in drug levels, consumption of drug by internalization of the complex rituximab-CD20, the pool of autoreactive B-cells that is in circulation available for drug action, drug wasting in urine through proteinuria and also epitope spreading.

Recognition and knowledge of some of these specific mechanisms of resistance has led to the use of other biologic agents. New monoclonal antibodies targeting CD20 have been developed and can be a rescue therapy for resistance PMN cases. However, as even these new-generation agents do not target memory plasma cells, therapies targeting these cells are promising. Inhibition of factors that activate autoreactive B-cells may also become an option. Additionally, a better understanding of the complement-mediated mechanisms of injury in PMN may bring to the pipeline novel biological therapies for this disease.

Key-words: Autoantibodies; glomerulonephritis, membranous; rituximab.

Primary membranous nephropathy (PMN) is the main cause of nephrotic syndrome in adults. The pathogenic knowledge gained in the past decade has placed PMN in the group of autoimmune diseases, which has dramatically changed diagnostic and therapeutic approaches. This kidney-specific disease is mediated by autoantibodies against podocyte membrane antigens, namely phospholipase $\mathrm{A} 2$ receptor (PLA2R), responsible for $52-78 \%$ of PMN cases ${ }^{1}$, and thrombospondin type-1 domain containing 7A (THSD7A), detected in 2-5\% of patients ${ }^{2}$.

The fact that approximately $20 \%$ of patients are negative for both antibodies makes it very likely that there are other culprit antigens for this disease. More recent studies have discovered other putative antigens.

Neural epidermal growth factor-like 1 (NELL-1) is a protein expressed mainly in kidney tubules. Sethi et al. ${ }^{3}$ showed that it was expressed along the glomerular basement membrane (GBM) in kidney biopsies from PLA2R-negative MN patients. NELL-1-associated MN is considered a unique kidney disease characterized by overexpression of NELL-1. Prevalence studies suggest that NELL-1 is the second most common antigen (after PLA2R) in PMN. Interestingly, 4 out of 5 patients with NELL-1 positive MN had an active malignancy, discovered at the time of or some months after MN diagnosis. More studies are needed to elucidate the association between NELL-1 MN and malignancy. One must keep in mind that even anti-PLA2R and THSD7A, the autoantibodies that have been highly studied so far, have been detected in patients with secondary MN, including malignancy-associated forms $\mathrm{s}^{4,5}$. Therefore, autoantibodies are a useful diagnostic tool in patients with MN but their positivity does not preclude the need to rule out secondary causes, such as malignancy. This is reinforced in the KDIGO clinical practice guidelines of glomerular diseases (public review draft) published in June $2020^{6}$.

The glycosyltransferases exostosin 1 (EXT1) and exostosin 2 (EXT2) have been recently identified by mass spectrometry studies in kidney biopsies from PLA2R/THSD7A double-negative patients ${ }^{7}$. Immunohistochemical study showed granular staining for EXT1 and EXT2 along the GBM, coincident with IgG granular staining. Also, $70.8 \%$ of the patients had abnormal autoimmune laboratory findings, such as positive anti-nuclear, anti-double stranded DNA, anti-SSA/SSB, or anti-ribonucleoprotein antibodies and 35\% had a clinical diagnosis of lupus. The authors suggested that EXT1/EXT2 may 
represent potential biomarkers or target antigens in secondary autoimmune MN.

A third new protein has been reported, Semaphorin 3B, that appears to be almost exclusive in pediatric patients ${ }^{8}$.

Treatment of PMN relies on optimal supportive care (SC), including blood pressure control, minimization of proteinuria through renin-angiotensin-aldosterone blockage, and control of nephrotic syndrome complications. In patients with persistent nephrotic syndrome, up to $40 \%$ will progress to kidney failure within 10 years $^{9}$. Immunosuppression therapy is therefore recommended for patients considered at risk of progressive kidney injury (persistent proteinuria, progressive decline in glomerular filtration rate (GFR) after 3-6 months of SC and/ or high titers of anti-PLA2R). Immunosuppression schemes commonly used are cyclophosphamide/steroids (modified Ponticelli), which is considered the first-line regimen in the 2012 KDIGO guidelines, calcineurin inhibitors (CNIs)/steroids, and more recently rituximab ${ }^{10}$. Rituximab can trigger B-cell death, and in consequence antibody production, by apoptosis, complement-mediated cytotoxicity, and antibody-dependent cellular cytotoxicity $(A D C C)^{11}$. B-cell therapy has gained ground as an option for $\mathrm{MN}$ in the last years, after some observational data ${ }^{12-15}$, but it was the MENTOR trial ${ }^{16}$ that placed rituximab as a viable candidate for first-line treatment. Fervenza et al. showed that this anti-CD20 agent was non-inferior to cyclosporin in inducing proteinuria remission at 12 -months, and was superior in maintaining proteinuria remission (60\% versus $20 \%$ clinical remission) up to 24 months, in patients at high risk of progressive disease. Additionally, the incidence of adverse events was similar in the two groups, but serious adverse events were more common in the cyclosporin group. The upcoming KDIGO clinical guidelines for glomerular diseases have already contemplated rituximab as a first-line option for MN, as an alternative for cyclophosphamide. However, in very high-risk patients, cyclophosphamide is still the preferred treatment ${ }^{6}$.

Despite the encouraging results of the MENTOR trial, one should not lose sight of the fact that $40 \%$ of patients receiving rituximab had treatment failure. The authors suggest that this high incidence of treatment failure may be overestimated due to the shorter follow-up period, arguing that proteinuria decline is gradual and the nadir may not be reached until 36 months after the initiation of treatment.

Noteworthy is the value of B-cell depletion monitoring, by CD19-cell counts, as a surrogate marker of rituximab response. This question hasn't been fully addressed as of yet but the KDIGO guidelines highlight that CD19 counts are not sufficient to judge rituximab efficacy ${ }^{6}$.

Notwithstanding, rituximab-resistant MN cases have been published ${ }^{17-19}$ and there is a need for alternative therapies for these patients. Rituximab resistance has been somewhat arbitrarily defined but is generally accepted as a lack of response or overt progression during or within 6 months of completion of a rituximab-containing regimen ${ }^{20}$. However, one should recognize that the persistence of proteinuria may not correspond to treatment resistance but, for instance, secondary segmental sclerosis development. Other clinical parameters, such as serum albumin, are important to the differential diagnosis and it may be reasonable to perform a second kidney biopsy.

\section{Table I}

Potential causes of rituximab-resistant primary membranous nephropathy

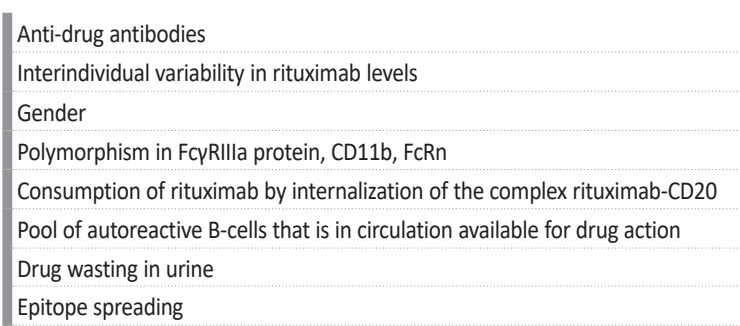

Several explanations have been postulated for truly rituximab-resistant cases (Table I).

The development of anti-drug antibodies is one of the proposed mechanisms. Rituximab is a chimeric monoclonal antibody including a human IgG1 constant region and a murine anti-human CD20 variable region that can elicit an immune response. Boyer-Suavet and colleagues ${ }^{18}$ found neutralizing anti-rituximab antibodies in 10 (22.7\%) out of 44 idiopathic membranous patients 6 months after one rituximab course ( 2 perfusions of $1 \mathrm{~g}$ at 2 -weeks interval). These patients had higher levels of CD19 counts at month 6, meaning faster B-cells reconstitution. Interestingly, remission rate was not different according to the presence or not of anti-rituximab antibodies, but relapses were more frequent in patients with anti-drug antibodies. These immunized patients have also required a higher number of rituximab infusions. In the future, we hope that anti-rituximab antibodies assays are available for daily clinical practice so we can tailor therapy.

Interindividual variability in rituximab levels is another factor that can explain differences in response to this agent. Most studies and published data refer to lymphoma and rheumatologic patients but one can extrapolate some findings to MN patients. Gender is a constitutive parameter that seems to influence drug pharmacokinetics, with men having a higher clearance of rituximab than women. Moreover, polymorphisms that are functionally relevant to the rituximab mode of action have been identified. There is a polymorphism described in the gene encoding FcyRIIla protein that alters its affinity for IgG1, thus diminishing affinity for rituximab. It is present in monocytes/macrophages and natural killer cells and is responsible for activation of $A D C C$, one of the mechanisms elicited by rituximab for inducing B-cells death. The genetic heterogeneity of CD11b, which plays a major role in rituximab's complement-enhanced ADCC (CR3$-A D C C)$, may also influence response to this drug. Also, the variability in the neonatal Fc receptor ( $\mathrm{FCRn}$ ), the endothelial cell receptor responsible for rituximab (and other drugs) recycling mechanism (Figure 1), may explain differences in rituximab response ${ }^{21}$. The internalization of the complex rituximab-CD20 is one possible explanation for resistance to rituximab over time (Figure 1). This process leads to degradation of the CD20-rituximab complexes and therefore less recruitment of macrophages and consequently less antibody-dependent phagocytosis $^{22}$. Some authors suggest that its efficacy can be enhanced by blocking the pathways involved in this process ${ }^{23}$. There is another factor that can influence response to anti-CD20 agents such as rituximab, which is the pool of autoreactive B-cells that is in circulation 


\section{Figure 1}

Rituximab mechanisms of internalization and recycling. Rituximab binds to the long loop of CD20 (transmembrane protein). The CD20-rituximab complex is then internalized. If rituximab binds to $\mathrm{FcRn}$ receptor, it is recycled back to the surface. If binding to FcRn does not occur, it is degraded in lysosome.

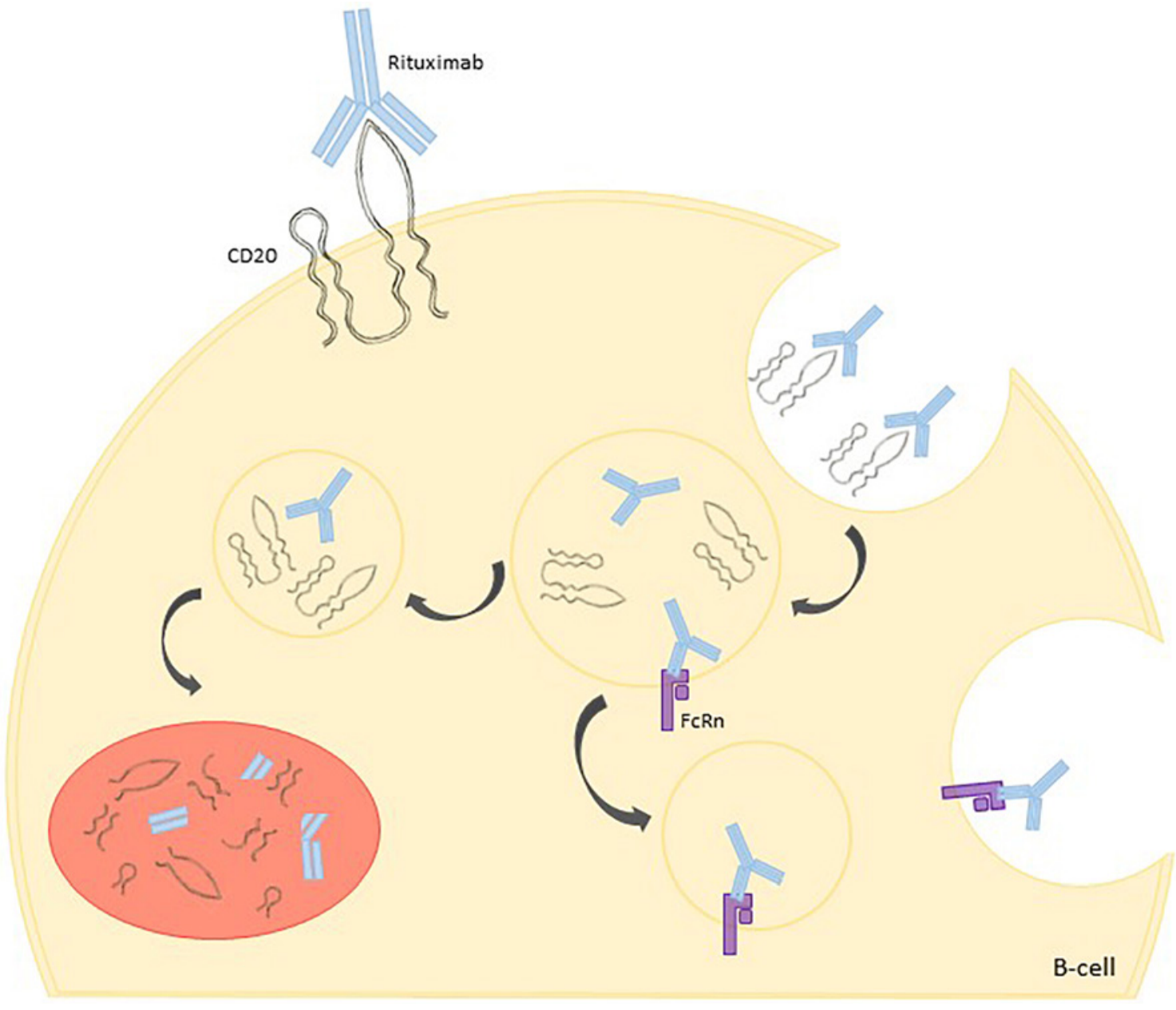

versus the pool that may chiefly reside in secondary lymphoid organs, thus being less available for drug action. An observation study (PEPTIDE, NCT04095156) is ongoing, aiming to analyze the circulating immune repertoire of $\mathrm{MN}$ patients before and after the infusion of B-cell lineage depleting agents, assessing the presence of circulating PLA2R autoreactive $B$ cells from appropriately stratified responder and non-responder patients. MN patients have an additional mechanism for lower rituximab levels related to proteinuria and drug wasting in urine ${ }^{24}$. Jacobs and colleagues ${ }^{25}$ evaluated urinary rituximab and total IgG levels in nephrotic patients previously treated with this agent. Urinary rituximab levels were detected in all patients, proving that measurement of urinary drug levels by flow cytometry is feasible. Moreover, the authors suggest that a correlation between total urinary IgG and rituximab levels may exist, but more studies are needed to elucidate this. In that case, the analysis of total IgG in urine, which can be easily performed in standard laboratories, might be sufficient to predict the probability of rituximab loss during treatment, and adjust posology.
Another explanation for the variable response to rituximab is related to epitope spreading. Epitope spreading is an immunologic phenomenon whereby an antibody or cellular response to a given antigen may extend from one location on the antigen (epitope) to involve other region(s) of the same antigen (intramolecular spreading) or adjacent antigens (intermolecular spreading) as the immune response mature ${ }^{26}$. In the case of PMN, autoantibodies firstly involved are typically directed to the cysteine-rich domain (CysR) of the PLA2R, and then extend to other epitopes on the same protein, commonly C-type lectin domains (CTLD) 1 and 7. Seitz-Polski et al.(27) showed that patients with anti-CyR-restricted activity had a better renal prognosis than patients that had epitope spreading beyond the CysR, who were less likely to achieve spontaneous remission. The same authors have shown that rituximab not only decreases anti-PLA2R titers but also reverses epitope spreading. However, to achieve this, it seems that higher doses of rituximab (two infusions of $1 \mathrm{~g} 2$-weeks apart versus four doses of $375 \mathrm{mg} / \mathrm{m}^{2}$ at 1- to 2 -week intervals) are needed ${ }^{11}$. This paper has highlighted the influence of epitope spreading on 
treatment resistance and suggested that a higher dose of rituximab is needed in spreaders.

Recognition and knowledge of some of these specific mechanisms of resistance has led to the development of other anti-B-cell agents (Table II). New monoclonal antibodies targeting CD20 are currently used in non-Hodgkin lymphomas and autoimmune diseases, including two humanized IgG1, obinutuzumab and ocrelizumab (Roche ${ }^{\circledR}$ ), and a fully-human $\operatorname{lgG1}$, ofatumumab $\left(\mathrm{GSK}^{\circledR}\right)$.

\section{Table II}

New potential biological treatments for primary membranous nephropathy

New anti-CD20 mAbs
Obinutuzumab - ongoing phase III double-blind RCT (REGENCY)
Ocrelizumab
Ofatumumab
Plasma-cell-depleting therapies
Anti-CD38 mAbs
Dataturumab
Isatuximib
MOR202 - ongoing phase Ib/Ila open-label clinical trial (M-PLACE)
Proteasome inhibitors
Bortezomib
Carfilzomib
Delanzomib
BLyS/BAAF inhibitor
Belimumab - ongoing double-blind RCT (REBOOT)

Legend: BLyS, B-lymphocyte stimulator; RCT, randomized clinical trial

Obinutuzumab is a humanized type II anti-CD20 monoclonal antibody (mAb). Type II mAbs, compared to type I, elicit little modulation of CD20 from the cell surface, thus having less consumption through internalization process, and have a higher affinity toward ADCC-induced death. The glycosylation of the molecule confers a better binding of effector immune cells and therefore higher ADCC and less dependence on $C D C$, compared to rituximab. Despite this theoretical advantage, the clinical benefits have been variable(28). It also induces a direct cell death mediated by lysosomes and less dependence on high levels of B-cell activating factor, which contributes to a greater depletion of memory $B$ cells that classically are more resistant to the effect of rituximab ${ }^{23}$. Obinutuzumab has already been successfully used in resistant MN cases. Klomjit et al. ${ }^{19}$ published a case report of 3 patients with PLA2R-associated MN that failed to respond to rituximab but who were successfully treated with this new anti-CD20, achieving partial clinical remission. Two of the three patients have also achieved immunologic remission and the third one, despite not fulfilling the restricted criteria of immunologic remission, had a dramatic decrease in anti-PLA2R titers. Moreover, the effect of obinutuzumab appears to be long-lasting, since one patient remained in clinical remission as long as 24 months after one course of the drug. There is an ongoing phase III double-blind randomized controlled trial (REGENCY, NCT04221477) assessing the efficacy, safety, and pharmacokinetics of obinutuzumab compared with placebo in patients with class III or IV lupus nephritis when added on to standard-of-care therapy consisting of mycophenolate mofetil and corticosteroids.
Ofatumumab binds to a different epitope than rituximab, as it can bind both the small and large extracellular loops of CD20, which may be the reason for increased complement-dependent cytotoxicity (CDC) activity compared to rituximab ${ }^{28}$. Moreover, it has a higher avidity for CD20 molecule comparing to rituximab, being able to induce cell death even in cells with a lower density of $\mathrm{CD} 20^{29}$. This latter mechanism is thought to be the reason for rituximab resistance in some hematologic malignancies. Because ofatumumab is fully-human, anaphylaxis is expected to be less common. This drug has shown efficacy in the treatment of B-cell lymphomas and other hematological malignancies which had previously not responded to rituximab. Ofatumumab has been used as a rescue therapy for patients with $\mathrm{MN}$ in whom retreatment with rituximab is contraindicated due to drug-related immune-complex mediated hypersensitivity reaction ${ }^{30}$. Podestà and colleagues ${ }^{31}$ published a case report of 3 patients in whom they tried ofatumumab and double-filtration plasmapheresis (DFPP) as a treatment for PMN. The best results were achieved with ofatumumab administration previous to DFPP, with a significant reduction in anti-PLA2R titers. This agent could be a safe and cost-effective rescue therapy for patients with $\mathrm{MN}$ sensitized against rituximab.

Whether new second and third generation anti-CD20 mAbs can achieve higher rates of sustained complete remission than rituximab remains to be determined, but such agents do not target long-lived memory plasma cells. In this field, plasma-cell-depleting therapies are promising. Plasma cells express CD38, which can be a target for anti-CD38 mAbs, such as dataturumab and isatuximib (table II). Less specific proteasome inhibitors (with anti-B and anti-T cell activities) such as bortezomib, and second-generation proteasome inhibitors with equal efficacy but improved safety profile, such as delanzomib and carfilzomib, are also options. Currently, published data regarding bortezomib usage in PMN is limited to a few case reports ${ }^{32,33}$. An open-label phase $\mathrm{lb} / \mathrm{ll}$ a clinical trial is currently running to characterize the safety and efficacy of the human anti-CD38 antibody MOR202 in patients with newly diagnosed, relapsed or refractory anti-PLA2R positive MN (M-PLACE, NCT04145440).

Another possible route for the treatment of $\mathrm{MN}$ patients is by inhibition of factors that activate autoreactive B cells (table II). BAFF (B cell-activating factor), also called B-lymphocyte stimulator (BLyS), and APRIL (a proliferation-inducing ligand) are members of the tumor necrosis factor (TNF) superfamily. Their main functions are to modulate survival and differentiation of B lymphocytes. A study has evaluated the relationship between these cytokines levels, anti-PLA2R titers, and clinical outcomes in PMN patients. They reported that anti-PLA2R positive patients had higher levels of BAFF and APRIL than negative patients. Cytokines levels decreased after 6 months of immunosuppressive therapy but the reduction was less pronounced in patients that were still anti-PLA2R positive at the end of the 6-month immunosuppression course. They suggest that serum levels of both BAFF/ BLyS and APRIL may have a role as predictors of anti-PLA2R seroconversion and good clinical outcomes in patients with PMN ${ }^{34}$. Belimumab, a human IgG1-k monoclonal antibody anti-BLyS (BAFF), has been approved for treatment of seropositive systemic lupus erythematosus patients and has been shown to reduce both disease activity and autoantibody levels ${ }^{35}$. An experimental study has demonstrated a reduction in proteinuria and circulating levels of anti-PLA2R by 86 and $97 \%$, respectively, in anti-PLA2R-positive PMN patients with 
nephrotic-range proteinuria, the effects continuing up to 2 years of treatment with belimumab ${ }^{36}$. The REBOOT trial (NCT03949855) may bring more data about this agent in NM.

Finally, significant complement activation is present in $\mathrm{MN}$ as evidenced by large spectral counts of complement proteins from C3- and C4-based pathways, including regulatory proteins of complement pathways. Ravindran et al. ${ }^{37}$ reported that the entire complement cascade is active, with both the classical/lectin and alternative pathways driving and contributing to activation of the terminal pathway. Another study in an experimental membranous nephropathy model revealed that the activation of the alternative pathway is essential for the development of proteinuria ${ }^{38}$. A better understanding of the complement-mediated mechanisms of injury in MN may help develop novel biological therapies for this disease.

In conclusion, a better understanding of the pathologic mechanisms involved in PMN has allowed the development of new therapeutic agents. Monoclonal antibodies are now part of the therapeutic armamentarium for PMN. Rituximab has emerged in recent years as a viable alternative to cyclophosphamide, albeit that this last agent is still the first-line option in very-high risk patients. With the wider use of this drug in PMN patients and a better understanding of its mechanism of action, rituximab limitations in some PMN patients have also emerged, propelling the search for new agents. These new drugs can overcome some of the rituximab limitations and ameliorate the prognosis of PMN patients.

\section{Disclosure of potential conflicts of interest: none declared}

\section{References}

1. Beck LHJ, Bonegio RGB, Lambeau G, Beck DM, Powell DW, Cummins TD, et al. M-type phospholipase A2 receptor as target antigen in idiopathic membranous nephropathy. N Engl J Med. 2009 Jul;361(1):11-21.

2. Tomas NM, Beck LHJ, Meyer-Schwesinger C, Seitz-Polski B, Ma H, Zahner G, et al. Thrombospondin type-1 domain-containing 7A in idiopathic membranous nephropathy. N Engl J Med. 2014 Dec;371(24):2277-2287.

3. Sethi S, Debiec H, Madden B, Charlesworth MC, Morelle J, Gross L, et al. Neural epidermal growth factor-like 1 protein (NELL-1) associated membranous nephropathy. Kidney Int [Internet] 2020;97(1):163-174. Available from: http://www.sciencedirect.com/science/article/pii/ S0085253819309950

4. Radice A, Pieruzzi F, Trezzi B, Ghiggeri G, Napodano P, D'Amico M, et al. Diagnostic specificity of autoantibodies to M-type phospholipase A2 receptor (PLA2R) in differentiating idiopathic membranous nephropathy (IMN) from secondary forms and other glomerular diseases. J Nephrol. 2018 Apr;31(2):271-278.

5. Hoxha E, Beck LH, Wiech T, Tomas NM, Probst C, Mindorf S, et al. An indirect immunofluorescence method facilitates detection of thrombospondin type 1 domain-containing 7A-specific antibodies in membranous nephropathy. J Am Soc Nephrol [Internet]. 2017 Feb 1;28(2):520-531. Available from: http://jasn.asnjournals.org/content/28/2/520.abstract

6. KDIGO. KDIGO clinical practice guideline on glomerular diseases (public review draft). 2020

7. Sethi S, Madden BJ, Debiec H, Charlesworth MC, Gross L, Ravindran A, et al. Exostosin 1/Exostosin 2-Associated Membranous Nephropathy. J Am Soc Nephrol. 2019 Jun;30(6):1123-1136.

8. Sethi S, Debiec H, Madden B, Vivarelli M, Charlesworth MC, Ravindran A, et al. Semaphorin 3B-associated membranous nephropathy is a distinct type of disease predominantly present in pediatric patients. Kidney Int. 2020 10:S0085-2538(20)30640-2;

9. Troyanov S, Wall CA, Miller JA, Scholey JW, Cattran DC. Idiopathic membranous nephropathy: definition and relevance of a partial remission. Kidney Int. 2004;66(3):1199-1205.

10. Couser WG. Primary membranous nephropathy. Clin J Am Soc Nephrol [Internet]. 2017;12(6):983997. Available from: http://cjasn.asnjournals.org/content/12/6/983.abstract

11. Seitz-Polski B, Dahan K, Debiec H, Rousseau A, Andreani M, Zaghrini C, et al. High-dose rituximab and early remission in PLA2R1-related membranous nephropathy. Clin J Am Soc Nephrol. 2019 Aug;14(8):1173-1182.

12. Fervenza FC, Abraham RS, Erickson SB, Irazabal MV, Eirin A, Specks U, et al. Rituximab therapy in idiopathic membranous nephropathy: a 2-year study. Clin J Am Soc Nephrol. 2010;5(12):2188-2198.

13. Ruggenenti P, Cravedi P, Chianca A, Perna A, Ruggiero B, Gaspari F, et al. Rituximab in idiopathic membranous nephropathy. J Am Soc Nephrol [Internet]. 2012 Aug 1;23(8):1416-1425. Available from: http://jasn.asnjournals.org/content/23/8/1416.abstract
14. Roccatello D, Sciascia S, Di Simone D, Solfietti L, Naretto C, Fenoglio R, et al. New insights into immune mechanisms underlying response to Rituximab in patients with membranous nephropathy: a prospective study and a review of the literature. Autoimmun Rev. 2016 Jun;15(6):529-538.

15. Bomback AS, Derebail VK, McGregor JG, Kshirsagar A V, Falk RJ, Nachman PH. Rituximab therapy for membranous nephropathy: a systematic review. Clin J Am Soc Nephrol. 2009 Apr;4(4):734-744.

16. Fervenza FC, Appel GB, Barbour SJ, Rovin BH, Lafayette RA, Aslam N, et al. Rituximab or cyclosporine in the treatment of membranous nephropathy. N Engl J Med [Internet]. 2019 Jul 3;381(1):3646. Available from: https://doi.org/10.1056/NEJMoa1814427

17. Podestà MA, Ruggiero $B$, Remuzzi $G$, Ruggenenti P. Ofatumumab for multirelapsing membranous nephropathy complicated by rituximab-induced serum-sickness. BMJ Case Rep [Internet]. 2020 Jan 1;13(1):e232896. Available from: http://casereports.bmj.com/content/13/1/e232896.abstract

18. Boyer-Suavet S, Andreani M, Lateb M, Savenkoff B, Brglez V, Benzaken S, et al. Neutralizing anti-rituximab antibodies and relapse in membranous nephropathy treated With rituximab. Front Immunol. 2019:10:3069.

19. Klomjit N, Fervenza FC, Zand L. Successful treatment of patients with refractory PLA2R-associated membranous nephropathy with obinutuzumab: a report of 3 cases. Am J Kidney Dis [Internet]. 2020 Jul 23; Available from: https://doi.org/10.1053/j.ajkd.2020.02.444

20. Rezvani AR, Maloney DG. Rituximab resistance. Best Pract Res Clin Haematol. 2011 Jun;24(2):203-216.

21. Cartron G, Trappe RU, Solal-Céligny P, Hallek M. Interindividual variability of response to rituximab: from biological origins to individualized therapies. Clin Cancer Res [Internet]. 2011 Jan 1;17(1):1930. Available from: http://clincancerres.aacrjournals.org/content/17/1/19.abstract

22. Mössner E, Brünker P, Moser S, Püntener U, Schmidt C, Herter S, et al. Increasing the efficacy of CD20 antibody therapy through the engineering of a new type II anti-CD20 antibody with enhanced direct and immune effector cell-mediated B-cell cytotoxicity. Blood. 2010 Jun;115(22):4393-4402.

23. Beers SA, French RR, Chan HTC, Lim SH, Jarrett TC, Vidal RM, et al. Antigenic modulation limits the efficacy of anti-CD20 antibodies: implications for antibody selection. Blood. 2010 un;115(25):5191-5201.

24. Boyer-Suavet S, Andreani M, Cremoni M, Brglez V, Benzaken S, Bernard G, et al. Rituximab bioavailability in primary membranous nephropathy. Nephrol Dial Transplant [Internet]. 2019 Mar 30;34(8):1423-1425. Available from: https://doi.org/10.1093/ndt/gfz041

25. Jacobs R, Langer-Jacobus T, Duong M, Stahl K, Haller H, Schmidt RE, et al. Detection and quantification of rituximab in the human urine. J Immunol Methods. 2017 Dec;451:118-121.

26. Salant DJ. Does epitope spreading influence responsiveness to rituximab in PLA2R-associated membranous nephropathy? Clinical journal of the American Society of Nephrology. Clin J Am Soc Nephrol. 2019;14(8):1122-1124

27. Seitz-Polski B, Dolla G, Payré C, Girard CA, Polidori J, Zorzi K, et al. Epitope spreading of autoantibody response to PLA2R associates with poor prognosis in membranous nephropathy. J Am Soc Nephrol. 2016 May;27(5):1517-1533.

28. Pierpont TM, Limper CB, Richards KL. Past, present, and future of rituximab - the world's first oncology monoclonal antibody therapy. Front Oncol. 2018;8:163.

29. Cheson BD. Ofatumumab, a novel anti-CD20 monoclonal antibody for the treatment of B-cell malignancies. J Clin Oncol Off J Am Soc Clin Oncol. 2010 Jul;28(21):3525-3530.

30. Podestà MA, Ruggiero B, Remuzzi G, Ruggenenti P. Ofatumumab for multirelapsing membranous nephropathy complicated by rituximab-induced serum-sickness. BMJ Case Rep. 2020;13(1):1-6.

31. Podestà MA, Gennarini $A$, Portalupi V, Rota $S$, Alessio MG, Remuzzi $G$, et al. Accelerating the depletion of circulating anti-phospholipase $A 2$ receptor antibodies in patients with severe membranous nephropathy: preliminary findings with double filtration plasmapheresis and ofatumumab. Nephron. 2020;144:30-35.

32. Hartono C, Chung M, Kuo SF, Seshan S V, Muthukumar T. Bortezomib therapy for nephrotic syndrome due to idiopathic membranous nephropathy. J Nephrol. 2014 Feb;27(1):103-106.

33. Barbari A, Chehadi R, Kfoury Assouf H, Kamel G, Jaafar M, Abdallah A, et al. Bortezomib as a novel approach to early recurrent membranous glomerulonephritis after kidney transplant refractory to combined conventional rituximab therapy. Exp Clin Transplant Off J Middle East Soc Organ Transplant. 2017 Jun;15(3):350-354.

34. Netti GS, Infante B, Spadaccino F, Godeas G, Corallo MG, Prisciandaro C, et al. Serum levels of BAFF and APRIL predict clinical response in anti-PLA2R-positive primary membranous nephropathy. Kolarz B, editor. J Immunol Res [Internet]. 2019;2019:8483650. Available from: https://doi. org/10.1155/2019/8483650

35. Navarra S V, Guzmán RM, Gallacher AE, Hall S, Levy RA, Jimenez RE, et al. Efficacy and safety of belimumab in patients with active systemic lupus erythematosus: a randomised, placebo-controlled, phase 3 trial. Lancet [Internet]. 2011 Feb 26;377(9767):721-731. Available from: https://doi.org/10.1016/S0140-6736(10)61354-2

36. Barrett C, Willcocks LC, Jones RB, Tarzi RM, Henderson RB, Cai G, et al. Effect of belimumab on proteinuria and anti-phospholipase $\mathrm{A} 2$ receptor autoantibody in primary membranous nephropthy. 2020;(June 2019):599-606.

37. Ravindran A, Madden B, Charlesworth MC, Sharma R, Sethi A, Debiec H, et al. Proteomic analysis of complement proteins in membranous nephropathy. Kidney Int Reports [Internet]. 2020;5(5):618626. Available from: http://www.sciencedirect.com/science/article/pii/\$2468024920300395

38. Luo W, Olaru F, Miner JH, Beck LH Jr, van der Vlag J, Thurman JM, Borza DB. Alternative Pathway Is Essential for Glomerular Complement Activation and Proteinuria in a Mouse Model of Membranous Nephropathy. Front Immunol. 2018:9:1433.

\section{Correspondence to:}

Ana Filipa da Silva Ferreira, MD

Nephrology department, Centro Hospitalar de São João

Alameda Prof. Hernâni Monteiro, 4200-319, Porto, Portugal

E-mail: filipasilvaf@gmail.com 